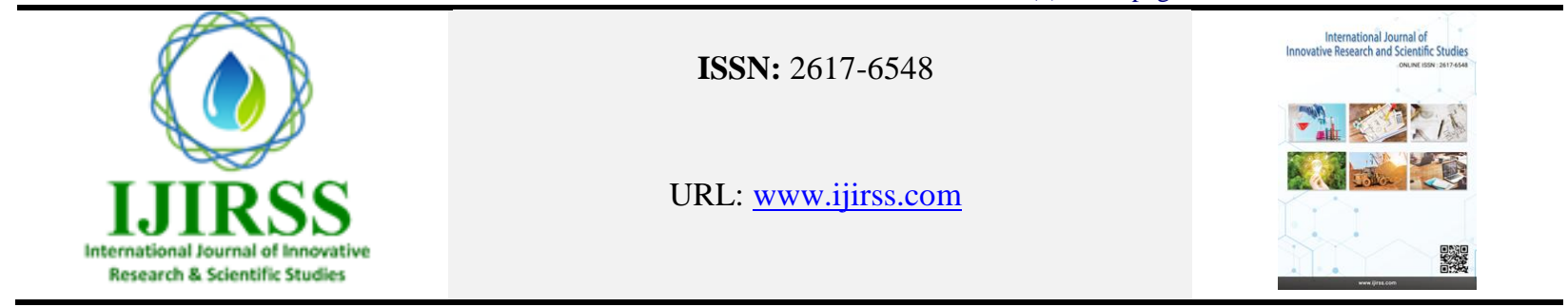

\title{
The Rate of Asymptomatic Throat Carriage of Streptococcus pyogenes and its Associated Risk Factors among Kabul University Students
}

\author{
Ahmad Zia Noori ${ }^{1}$, Haji Mohammad Naimi ${ }^{2 *}$, Hashmatullah Yousufi ${ }^{3}$ \\ ${ }^{1,2,3}$ Department of Microbiology, Faculty of Pharmacy, Kabul University, Kabul, Afghanistan \\ *Corresponding author: Haji Mohammad Naimi (hm_naimi@yahoo.com)
}

\begin{abstract}
Streptococcus pyogenes (S. pyogenes) is the main agent of acute pharyngitis and skin infections that may result in the late complications of glomerulonephritis and rheumatic fever. Infection with streptococcus group A is a global health problem, which is most common in children and adults. This study was conducted to investigate the rate of $S$. pyogenes throat carriers and its main risk factors among healthy students of Kabul university. In the present study pharyngeal swabs of 260, [155 (59.6\%) were male and $105(40.4 \%)$ were female] asymptomatic university students aged between 19-30 years, were collected and immediately transported to the laboratory for detection of $S$. pyogenes following standard microbiological procedures. Production of beta hemolytic colonies on blood agar, sensitivity to bacitracin antibiotic, gram stain positivity, catalase negativity test and streptococcal grouping latex kit (ProlexTM) tests were used to identify and differentiate $S$. pyogenes from other streptococcus spp. Statistical analysis of data was performed using SPSS 21, Chi-square and Logistic regression tests were applied for the categorical data analysis. A P value equal to or less than 0.05 was considered statistically significant. Totally $61(23.5 \%)$ beta hemolytic streptococci were isolated from 260 samples. Among 61 beta hemolytic isolates, $44(16.9 \%)$ were identified as S. pyogenes. The colonization rate of S. pyogenes was higher in male 25 $(56.8 \%)$ than female $19(43.2 \%)$, which was not statistically significant $(\mathrm{p}=0.678)$. Age, residence of the students at hostel and shared utensil use were not statistically significant $(\mathrm{p}=0.088, \mathrm{p}=0.449, \mathrm{p}=0.241$ respectively), but the number of children in the family was an important risk factor. People with 1-3 children had a 23 -fold higher risk $(\mathrm{p}<0.05)$, and people with 4-6 children had a 27-fold higher risk of carrying $S$. pyogenes, than those who did not had any children $(\mathrm{p}<0.05)$. In the present study the asymptomatic throat carriage rate of S. pyogenes among Kabul University students, was high. Among all risk factors the number of children in the family was significantly associated with $S$. pyogenes throat carriage.
\end{abstract}

Keywords: Streptococcus pyogenes, Pharyngeal Swab, University Students.

DOI: 10.53894 /ijirss.v3i4.48

Funding: This study received no specific financial support.

History: Received: 23 September 2020/Revised: 6 November 2020/Accepted: 4 December 2020/Published: 11 December 2020

Licensed: This work is licensed under a Creative Commons Attribution 4.0 License $($ (c) $)$ EY

Acknowledgement: Authors thank the laboratory of Microbiology of the faculty of Pharmacy of Kabul University for providing them the materials and reagents for conducting this research.

Competing Interests: The authors declare that they have no conflict of interests.

Transparency: The authors confirm that the manuscript is an honest, accurate, and transparent account of the study was reported; that no vital features of the study have been omitted; and that any discrepancies from the study as planned have been explained.

Ethical: This study follows all ethical practices during writing. 


\section{Introduction}

S. pyogenes is a beta hemolytic, gram positive and aerotolerant bacteria which cause sore throat and multiple skin infections. On the basis of Lancefield classification these bacteria belong to group A streptococci, which is called Group A beta-hemolytic streptococci (GABHS) [1]. S. pyogenes among other streptococci is the major human pathogen which causes several infections varied from mild including skin and throat infections to sever life-threatening conditions such as Rheumatic fever, acute glomerulonephritis and septicemia [2,3]. The involvement of this species in severe disease is partly due to the substantial array of surface antigens, toxins and enzymes it generates [4].

Almost all individuals can asymptomatically carry this organism, but its prevalence is higher in overcrowding conditions, as its interpersonal dissemination occurs though sputter or by direct contact, mainly in the environments where large crowds get together, such as schools, day care centers and universities [5]. People who asymptomatically carry this organism on their pharynx could play a role in the spread of infection (source of bacterial dissemination) to households and community settings such as universities, school, day care center and office $[3,6,7]$. Evidence shows that $S$. pyogenes asymptomatic throat carriage could also be a risk factor, which can cause infections and may lead to life-threatening conditions such as rheumatic fever, rheumatic heart disease and acute glomerulonephritis [8]. Although, these conditions are not common in developed countries, but in most developing countries it remains the leading cause of acquired health disease [9]. Although the throat carriage rate of this organism may vary with the season of the year, personal hygiene, geographic location and economic situation. Most of research conducted on the throat carriage rate of $S$. pyogenes, showed that age, gender, family size, shared use of utensils and hostel are the main risk factors [10]. In this study we investigate the rate of asymptomatic carriage of $\beta$-hemolytic streptococci, specially the presence of $S$. pyogenes in the oropharynx and its related risk factors among healthy students of Kabul University.

\section{Methods and Materials}

In this study a total of 260 asymptomatic university students 151 males (58.1\%) and 109 females (41.9\%) were included after informed consent. The age of the students ranged between $19-30$ years (mean 22.02 years). All participants were questioned before sample collection in regard to the demographic profile and presence of $S$. pyogenes related clinical symptoms such as sore throat, fever, malaise and chills, and also each of them was physically examined for the presence of related signs at their pharynx. All those students, who had any such signs or symptoms were excluded.

Pharyngeal swabs of 260 asymptomatic university students were collected and immediately transported to the laboratory for detection of $S$. pyogenes following standard microbiological procedures [11-13]. Collected samples were immediately inoculated on 5\% sheep blood agar CNA (blood agar with colistin and nalidicxic acid) and incubated at $37 \mathrm{C}^{\circ}$ for $24-48 \mathrm{~h}$ with $5 \% \mathrm{CO}_{2}$ atmosphere to detect $\beta$-hemolytic colonies. All beta hemolytic colonies were sub-cultured on blood agar and a bacitracin disc ( $0.05 \mathrm{U}$, Oxoid) was used to differentiate $S$. pyogenes form other $\beta$-hemolytic streptococcus [11-13].

Suspected colonies with $\beta$-hemolytic reaction on blood agar and presence of inhibition zone around the disk were subjected for catalase test, gram stain and streptococcal grouping latex kit (prolexTM). Positive and negative reactions of each test was registered, data entry and analysis were done using SPSS version 21 software. Comparisons between different variables were made using Chi-square and logistic regression tests.

\section{Results}

A total of 260 asymptomatic university students with the mean age of 22 (range 19-30) years, were included in this research. Out of 260 students, $155(59.6 \%)$ were male and $105(40.4 \%)$ were female. Altogether $61(23.5 \%)$ beta hemolytic streptococci were isolated from 260 samples. Out of 61 beta hemolytic isolates, 44 were identified as $S$. pyogenes (Figure $1)$.

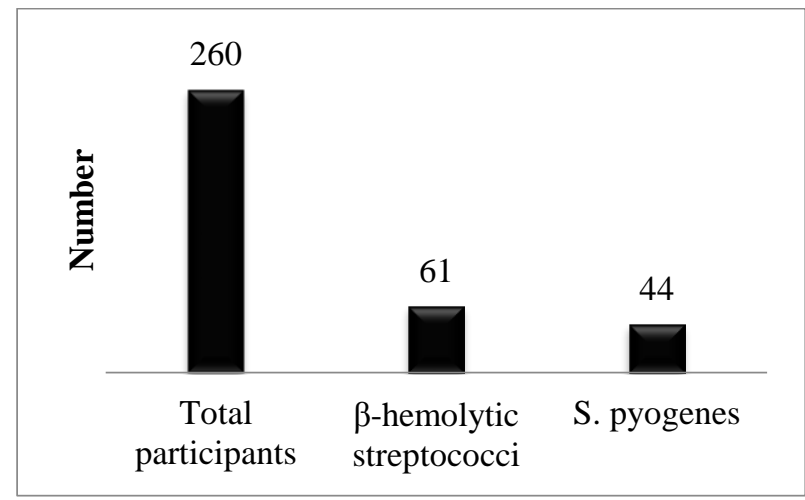

Figure-1.

The number of participants which carry $S$. pyogenes and other beta hemolytic streptococci asymptomatically.

The carrier rate of $S$. pyogenes among university student was $16.9 \%$. According to gender the carrier rate of $S$. pyogenes was higher in male, compared to female ( $56.8 \%$ vs $43.2 \%$ respectively), but this difference was not statistically significant $(\mathrm{p}=0.678)$ (Table 1). There was no significance difference between students living in the hostel and students 
living with their family ( $\mathrm{p}=0.449$ ) (Table 1 ). The ages of students in this study ranged from 19-30 years (which divided into four groups of 19-21, 22-24, 23-27 and 28-30 years), the highest number of asymptomatic S. pyogenes carrier was found in age group of 19-21 years, however this difference was not statistically significant $(\mathrm{p}=0.088)$, but the result shows that the prevalence of $S$. pyogenes decreased by age (Table 2). Shared utensil use between family members also was not statistically significant $(\mathrm{p}=0.241)$. In this study the number of children in the family was an important risk factor. Students with $1-3$ children in the family had a 23 -fold higher risk of carrying $S$. pyogenes than those who $(\mathrm{p}<0.05)$, and those students who had 4-6 children in their family, had a 27-fold higher risk of carrying $S$. pyogenes on their throat than those didn't had any children $(\mathrm{p}<0.05)$ (Table 3$)$.

Table-1.

Comparison of S. pyogenes carriage based on gender and living in hostel.

\begin{tabular}{|c|c|c|c|c|c|c|c|}
\hline S. pyogenes carriage & Male & Female & $\mathbf{P}$ & Hostel & Yes & No & $\mathbf{P}$ \\
\hline No of carriers & 25 & 19 & \multirow{3}{*}{0.678} & No of carriers & 11 & 33 & \multirow{3}{*}{0.449} \\
\hline No of non-carriers & 155 & 105 & & No of non-carriers & 54 & 206 & \\
\hline Total & 180 & 124 & & Total & 65 & 239 & \\
\hline
\end{tabular}

Table-2.

Comparison of $S$. pyogenes carriage, based on age

\begin{tabular}{l|l|l|l|l|l|l|l|l|l|l|l|l|l}
\hline Age/year & $\mathbf{1 9}$ & $\mathbf{2 0}$ & $\mathbf{2 1}$ & $\mathbf{2 2}$ & $\mathbf{2 3}$ & $\mathbf{2 4}$ & $\mathbf{2 5}$ & $\mathbf{2 6}$ & $\mathbf{2 7}$ & $\mathbf{2 8}$ & $\mathbf{2 9}$ & $\mathbf{3 0}$ & $\mathbf{P}$ \\
\hline $\begin{array}{l}\text { No of S. } \\
\text { pyogenes carriers }\end{array}$ & 6 & 5 & 13 & 9 & 4 & 4 & 3 & 0 & 0 & 0 & 0 & 0 & \\
\hline $\begin{array}{l}\text { No of non- } \\
\text { carriers }\end{array}$ & 10 & 39 & 48 & 40 & 35 & 17 & 12 & 6 & 5 & 3 & 0 & 1 & 0.088 \\
\hline Total & 16 & 44 & 61 & 49 & 39 & 21 & 15 & 6 & 5 & 3 & 0 & 1 & \\
\hline
\end{tabular}

Table-3.

Comparison of $S$. pyogenes carriage based on number of children in the family.

\begin{tabular}{l|l|l|l|l|l|l|l|l|l|l|l|l|l} 
Comparison of $S$. pyogenes carriage based on number of children in the family. & $\mathbf{0}$ & $\mathbf{1}$ & $\mathbf{2}$ & $\mathbf{3}$ & $\mathbf{4}$ & $\mathbf{5}$ & $\mathbf{6}$ & $\mathbf{7}$ & $\mathbf{8}$ & $\mathbf{9}$ & $\mathbf{1 0}$ & $\mathbf{1 3}$ & $\mathbf{P}$ \\
\hline No of children & 2 & 2 & 12 & 11 & 8 & 3 & 3 & 2 & 0 & 0 & 0 & 1 & \\
\hline No of S. pyogenes carriers & 59 & 66 & 53 & 29 & 4 & 2 & 2 & 1 & 0 & 0 & 0 & 0 \\
\hline No of non-carriers & 61 & 68 & 65 & 40 & 12 & 5 & 5 & 3 & 0 & 0 & 0 & 1 & \\
\hline Total & 6 &
\end{tabular}

\section{Discussion}

There is no previous study regarding asymptomatic carriage of $S$. pyogenes in Afghanistan. Our study showed that $S$. pyogenes was the predominant organism among beta hemolytic streptococci in asymptomatic university students. In the present study we found that $23.5 \%$ (61/260) of students ages 19-30 were beta hemolytic streptococci carrier. Out of 61 beta hemolytic streptococci carrier, $72.1 \%$ (44/61) of students had S. pyogenes on their throat (carrier state). The prevalence (throat carrier rate) of $S$. pyogenes in Kabul university students was $16.9 \%$ compared with India 11.2 to 34\% [14] Turkey 2$46 \%$ [15] Argentine 5-7\% [16]. According to ages, the highest prevalence of $S$. pyogenes asymptomatic throat carriage was in the lowest age sub group (19-21 years) compared to other sub groups (22-24, 25-27 and 28-30y), which shows that throat carriage of this organism decrease by age and it was similar with the many previous studies in other countries [6, 17-20]. In our study the number of children in the family (large family size) was an important risk factor. Students with 1-3 children in the family had a 23-fold higher risk of carrying $S$. pyogenes, and students with 4-6 children in their family, had a 27-fold higher risk of carrying $S$. pyogenes than those who did not had any children. This finding was also similar to the previous study which was conducted in Nepal [20]. The carrier rate of S. pyogenes according to gender was not statistically significant. Most of studies conducted in this area also showed that the carrier rate for both male and female were similar $[17,21]$.

\section{Conclusion}

In the present study the asymptomatic throat carriage rate of $S$. pyogenes among Kabul university students, was $16.9 \%$. Among all risk factors higher number of children in the family was significantly associated with asymptomatic carriage of S. pyogenes in throat. As it was first study conducted only among Kabul University students, therefore cannot be representative of $S$. pyogenes carrier rate among healthy individuals in Afghanistan. further studies are needed to study the prevalence of $S$. pyogenes among healthy individuals and associated risk factors other than university students to find more accurate results and establish prevention protocols to people who are at risk of acquisition and development of disease and complications.

\section{References}

[1] A. Manandhar, Y. Shah, and J. Shrestha, "Study on the prevalence of beta haemolytic streptococcus among school children," J. Nepal Paediatr, Soc, vol. 33, pp. 45-47, 2013. 
[2] A. Asrat, B. Getenet, S. Zewdineh, and D. Deresse, "Asymptomatic pharyngeal carriage rate of Streptococcus pyogenes, its associated factors and antibiotic susceptibility pattern among school children in Hawassa town, southern Ethiopia," BMC Res. Notes vol. 12, pp. 1-6, 2019. Available at: https://doi.org/10.1186/s13104-019-4601-9.

[3] S. S. Ashgar, B. Abeer, A. Johargy, H. M. El-Said, M. H. Mukhtar, and A. A. Saati, "Prevalence of Streptococcus pyogenes among pre-school children ages 4 to 6 in Makah city, Saudi Arabia. Glo. Adv. Res," Journal of Medical Sciences, vol. 6, pp. 08-13, 2017.

[4] K. P. Talaro, Foundations in microbiology vol. 4. New York: McGraw-Hill, 2002.

[5] V. M. Morais, "Prevalence of B-hemolytic streptococcus in children with special health care needs," Braz J Otorhinolaryngol, pp. 110-115, 2012.

[6] S. Haddad, M. IIktac, S. Ogmen, and G. Celik, "Investigation of Streptococcus pyogenes carriage among pharmacy students in north cyprus," EMU J. Pharm Sci, pp. 1-6, 2019.

[7] F. Nabipour and M. Tayarzadeh, "Prevalence of beta hemolytic Streptococcus carrier state and its sensitivity to different antibiotics among guidance school children in Kerman," Iran. Amer. J. Infect Dis, vol. 1, pp. 128-131, 2005.

[8] A. C. Charmaine, S. E. Jacob, and T. Menon, "Pharyngeal carriage of group A streptococci in school children in chennai," Indian J. Med. Res, vol. 124, pp. 195-198, 2006.

[9] J. R. Carapetis, A. C. Steer, E. K. Mulholland, and M. Weber, "The global burden of group a streptococcal diseases," Lancet infect. Dis, vol. 5, pp. 685-694, 2005. Available at: https://doi.org/10.1016/s1473-3099(05)70267-x.

[10] N. Principi, P. Marchisio, G. C. Schito, and S. Mannelli, "Risk factors for carriage of respiratory pathogens in the nasopharynx of healthy children," The Pediatric Infectious Disease Journal, vol. 18, pp. 517-523, 1999. Available at: https://doi.org/10.1097/00006454-199906000-00008.

[11] J. Vandepitte, J. Engbaek, K. Rohner, P. Piot, P. Heuck, and C. Claus, "Basic laboratory procedures in clinical bacteriology," 2nd ed Geneva: Switzerland, WHO, 2003, pp. 1-188.

[12] B. Spellerberg, C. Brandt, J. H. Jorgensen, M. A. Pfaller, K. C. Carrol, M. L. Landry, G. Funke, S. S. Richter, and D. W. Warnock, "Manual of clinical microbiology," pp. 383-402, 2015.

[13] A. L. Bisno, M. A. Gerber, J. M. Gwaltney, J. L. E.L. Kaplan, and R. H. Schwartz, "Practice guidelines for the diagnosis and management of group A," Streptococcal Pharyngitis, Infectious Diseases Society of America, pp. 113-125, 2002.

[14] A. Mukundan and S. Vijayakumar, "Pharyngeal carriage of group a streptococci among school children," Journal of International Medicine and Dentistry, vol. 4, pp. 18-26, 2017. Available at: https://doi.org/10.18320/jimd/201704.0118.

[15] S. Metintas, C. Kalyoncu, S. Etiz, N. Kiraz, and N. Unsal, "Prevalence of group A beta haemolytic Streptococcus carriers in primary school students of cifteler, Turkey," Anatolia Med J, vol. 13, pp. 17-27, 1991.

[16] National Committee of Pediatric Infectology, "Blue book of pediatric infectology," Buenos Aires: Argentine Society of Pediatrics, pp. 513-515, 1988.

[17] A. Abdissa, D. Asrat, G. Kronvall, B. Shitu, D. Achiko, M. Zeidan, L. K. Yamuah, and A. Aseffa, "Throat carriage rate and antimicrobial susceptibility pattern of group A streptococcus (GAS) in healthy Ethiopian school children," Ethiop med Journal, pp. 125-130, 2011.

[18] G. Delpech, M. Sparo, B. Baldaccini, G. Pourcel, S. Lissarrague, and L. G. Allende, "Throat carriage rate and antimicrobial resistance of Streptococcus pyogenes in rural children in argentina," Journal of Preventive Medicine and Public Health, vol. 50, pp. 127-132, 2017.

[19] J. M. Martin, M. Green, K. A. Barbadora, and E. R. Wald, "Group A Streptococci among school-aged children: clinical characteristics and the carrier state," American Academy of Pediatrics, pp. 1212-1219, 2004.

[20] A. Prajapati, S. K. Rai, R. K. Mukhiya, and A. B. Karki, "Study on carrier rate of Streptococcus pyogenes among the school children and antimicrobial susceptibility pattern of isolates," Nepal Med Call Journal, pp. 169-171, 2012.

[21] R. Durmaz, B. Durmaz, M. Bayraktar, I. H. Ozerol, M. T. Kalcioglu, E. Aktas, and Z. Cizmeci, "Prevalence of group A Streptococcal carriers in asymptomatic children and clonal relatedness among isolates in Malatya, Turkey," Journal of clinical microbiology, vol. 41, pp. 5285-5287, 2003. 